\title{
How Can Practice-based Research Contribute to the Elimination of Health Disparities?
}

\author{
George Rust, MD, MPH, and Lisa A. Cooper, MD, MPH
}

Racial, ethnic, and socioeconomic disparities in health care and health outcomes are well documented. Disparities research is evolving from documenting these disparities, to understanding their causes and mechanisms, and finally to conducting interventional research to reduce or eliminate disparities. Unfortunately, few studies to date have demonstrated substantial reductions in health outcomes disparities.

Traditional experimental models of research that test a single intervention held constant throughout the study period may not have the power to impact complex clusters of comorbid health disparities in patients who receive care in underresourced primary care safety net practice settings. New models of research will be required to test dynamic, multidimensional interventions that triangulate on patients, providers, and communities and are continuously improved with every radar-sweep of feedback from rapid-cycle measurement of population health outcomes on a community-wide basis. In this article, we review 12 promising strategies that could substantially increase the impact of research on eliminating health disparities in America. (J Am Board Fam Med 2007;20:105-114.)

Health disparities are pervasive and persistent. Several decades of research have documented disparities in care and outcomes across a wide variety of clinical conditions. ${ }^{1-3}$ Health disparities have been documented between racial/ethnic groups, rural/ urban and other geographic settings, as well as for socioeconomic status and for the uninsured. ${ }^{4}$ The most pervasive and deadly health disparities in the United States have been documented along racial lines. More than 80,000 lives each year could be saved just by eliminating the black-white mortality gap in America. ${ }^{5}$ Racial and ethnic disparities have been found across all age/gender groups and geo-

This article was externally peer-reviewed.

Submitted 6 August 2006; revised 17 October 2006; accepted 23 October 2006.

From the National Center for Primary Care, Morehouse School of Medicine, Atlanta, GA (GR); and Welch Center for Prevention, Epidemiology, and Clinical Research, Johns Hopkins Medical Institutions, Baltimore, MD (LAC).

Funding: LAC is funded by grants from the Agency for Healthcare Research and Quality (R01 HS13645) and the National Heart, Lung, and Blood Institute (1R01HL69403 and K24HL083113).

Previous presentation: This article is based on a presentation made at the 2006 Agency for Healthcare Research and Quality National Practice-based Research Network Conference, Bethesda, MD, May 15-17, 2006.

Conflict of interest: none declared.

Corresponding author: George Rust, MD, MPH, National Center for Primary Care, Morehouse School of Medicine, 720 Westview Drive, Atlanta, GA 30310 (E-mail: GRust@msm.edu). graphic regions, in all health care settings, at all levels of care, and across all dimensions of quality. ${ }^{6}$ When poverty, non-white race, non-English language, lack of insurance, and underserved community all coexist, the inequities are compounded. This creates a continuum of disparities beyond just race, ranging from low-disparity populations (upper-income, white, suburban, well educated, and insured) to high-disparity populations (low-income, uninsured, minority race, and rural or innercity community).

Although the existence of health disparities has been proven, the job of documenting disparities is not finished. More frequent measurement of disparities could provide ongoing monitoring of progress at the national level, and more microsurveillance could document disparities at state and local levels. ${ }^{7}$ The biggest gap in disparities research, however, is that very few interventional studies have demonstrated significant reductions in health disparities. The primary focus of disparities research must now be to test interventions that will actually reduce or eliminate health disparities.

Although there is a robust literature of research on quality improvement and health promotion, none of this research to date has had a significant impact on reducing health disparities. Therefore, we sought to identify from the literature some of the research strategies that might have greater po- 


\begin{tabular}{|c|c|}
\hline Why Current Disparities Research Sometimes Falls Short & How Practice-based Research Could Meet the Need \\
\hline 1. Research in academic or closed-panel settings & $\begin{array}{l}\text { Conduct research in real-world, limited resource, } \\
\text { high-disparity primary care practice settings }\end{array}$ \\
\hline 2. Nondiverse research teams & $\begin{array}{l}\text { Develop diverse research teams that are proportionately } \\
\text { representative of the disparity population being studied }\end{array}$ \\
\hline 3. Investigator-initiated research & True community partnership \\
\hline 4. Focus on changing provider behaviors & $\begin{array}{l}\text { Research on systems change involving patients, teams, and } \\
\text { processes of care }\end{array}$ \\
\hline 5. 'Inside-the-practice' research & $\begin{array}{l}\text { Blur the boundaries between practice-based research and } \\
\text { community-based interventions }\end{array}$ \\
\hline 6. Focus on process measures & $\begin{array}{l}\text { Measure health outcomes at the community population } \\
\text { health level }\end{array}$ \\
\hline 7. Narrowly focused single disease interventions & $\begin{array}{l}\text { Address complex mix of disparities in chronic disease } \\
\text { outcomes, risk factors, and mental health co-morbidities }\end{array}$ \\
\hline 8. Experiments test one intervention & $\begin{array}{l}\text { Test multidimensional interventions that triangulate on } \\
\text { improved outcomes from at least three } \\
\text { directions-provider, patient, and community }\end{array}$ \\
\hline $\begin{array}{l}\text { 9. Static interventions held constant throughout the } \\
\text { study period }\end{array}$ & Test dynamic, constantly-improving interventions \\
\hline 10. Academic cycle time & $\begin{array}{l}\text { Rapid-change cycles, continuously revising intervention } \\
\text { based on rapid-feedback health outcomes data loops }\end{array}$ \\
\hline 11. Randomized-controlled clinical trials & $\begin{array}{l}\text { Alternative study designs to measure multidimensional, } \\
\text { dynamic interventions repeatedly }\end{array}$ \\
\hline 12. Replicability without scalability & $\begin{array}{l}\text { Test interventions that are both replicable and scalable in } \\
\text { real-world, underresourced settings that serve } \\
\text { high-disparity populations }\end{array}$ \\
\hline
\end{tabular}

tential to move the needle on eliminating health disparities, with an emphasis on real-world, community-level, or practice-based research in primary care settings serving high-disparity populations. High-disparity populations (ie, low-income, uninsured, and/or minority populations suffering disproportionate morbidity and mortality because of health disparities) often receive care in safety net settings. In fact, there is some evidence to suggest that practices proactively serving high-disparity segments of the population (eg, community health centers and Veteran's Health Administration sites) actually achieve lower disparities or near-equal care across black/white and uninsured/insured strata of their patients, even though they both serve disproportionately low-income and minority (ie, highdisparity) populations. ${ }^{8-11}$ Unfortunately, when care received both inside and outside the Veteran's system (ie, Veteran's Health Administration plus Medicare) is measured, disparities reemerge. ${ }^{12,13}$ What is lacking are studies demonstrating the effectiveness of an explicit intervention designed to eliminate an established baseline disparity within these or other practice settings.

Mold and Peterson have described primary care practice-based research networks as working at the interface between research and quality improvement. ${ }^{14}$ The National Institutes of Health (NIH) Roadmap, first unveiled by NIH Director Elias Zerhouni in 2003, has provided the opportunity for primary care practice-based research to play a central role in the "transformative" new translational strategies envisioned by the roadmap. ${ }^{15}$ In the same way, practice-based research in primary care settings that serve high-disparity patient populations provide an essential interface between research and eliminating health disparities.

Here are 12 potential strategies that could help primary care researchers to move the needle on health disparities. These are listed in Table 1, where they are contrasted with characteristics of more traditional research models.

\section{Translation-Squared in Real World, High- Disparity Settings}

The first gap that practice-based research can address is the need to conduct disparities research in the settings that serve high-disparity communities (rather than resource-enhanced academic settings or closed-panel HMOs). A translation-squared research strategy applies interventions to high-disparity patient populations in underresourced safety 
net health centers, under real-world constraints experienced by their primary care clinicians. ${ }^{16}$ Translational research has traditionally taken discoveries from the bench to the bedside, but the new challenge is to take research out to the curbside and the countryside. ${ }^{17}$ Conducting research in these settings generates more unique challenges than in the academic environment and requires different resources, staffing, and skill sets. ${ }^{18}$

Unfortunately, most NIH-funded research is not conducted by primary care generalists, ${ }^{19}$ and most quality improvement interventions are not specifically designed for high-disparity populations. In a recent systematic review of quality improvement intervention trials that included mostly ethnic minority patients, researchers found that most interventions did not specifically target the needs of minority patients, and the studies did not address which strategies are most effective in reducing disparities between minority and white patients. ${ }^{20}$ The review suggested distinguishing between interventions targeting overall quality of care versus interventions specifically targeting disparities in care for racial/ethnic minority populations (such as reducing provider bias or improving intercultural communication skills).

\section{Diverse Teams}

A second deficit that practice-based research could address is the relative lack of underrepresented minority investigators on most research teams. Disparities research conducted by nondiverse teams of investigators at majority-dominant institutions may have unconscious bias or blinders in its design (who asks the research question?) or its interpretation (who says what the results mean?). For many years, the National Household Survey of Drug Abuse was cited as showing African Americans at high risk for crack cocaine use. In 1993, JAMA published LillieBlanton's reanalysis of precisely the same database in which she added one covariate that completely changed the conclusions. ${ }^{21}$ Whereas previous studies had controlled for individual education and socioeconomic status, the authors added neighborhood characteristics to the multivariate analysis, and the racial differences disappeared. Given a poor neighborhood, whites were at least as likely as persons of color to use crack cocaine. The original analyses had the effect of tagging a racial group as high risk for a negative behavior. By asking a more nuanced research question, Lillie-Blanton identi- fied a variable (neighborhood poverty) which could potentially be improved by intervention, dramatically changing the policy implications.

Diverse teams with investigators who are racially and culturally representative of the populations being studied may better capture the subtle nuances of race, ethnicity, culture, poverty, health, and resiliency. However, less than $1 \%$ of NIH research grant dollars are currently funding underrepresented minority investigators. ${ }^{22,23}$ Although nearly $26 \%$ of the US population is composed of African American, American Indian, Hispanic, or Latino people, ${ }^{24}$ only $6.4 \%$ of US physicians and $7.2 \%$ of US medical school faculty are from these groups. Most of the underrepresented minority faculty are at lower academic ranks such as clinical instructors. $^{25,26}$ In contrast, clinicians practicing in safety net settings that serve minority populations are significantly more likely to be representative of the populations they serve. ${ }^{27}$ Therefore, participation of these clinicians in practice-based research networks can substantially add diversity to the disparities research team.

\section{In Partnership with Communities}

In the words of NIH Director Elias Zerhouni, "as disease burden has shifted from acute conditions to chronic conditions primarily seen in community rather than tertiary centers, new approaches for forging relationships with local and regional community partners will become increasingly critical. ${ }^{28}$ In other words, research must be conducted not only in community settings but in partnership with communities. Communities are not laboratories. Disparities research must be conducted in partnership with communities to ensure that the interventions are community-responsive and that the interpretation of results resonates with the community's understanding of what the data mean. This implies bidirectional communication, treating community-based practitioners and their patients as the true experts in local realities, involving them and their communities in the development of culturally relevant solutions and interventions. Wallerstein and Duran have described this partnership by defining community-based participatory research as "an orientation to research that focuses on relationships between academic and community partners, with principles of colearning, mutual benefit, and long-term commitment and incorporates community theories, participation, and practices 
into the research efforts." ${ }^{29}$ There also is often a profound mismatch in resources and power between the research institution (even smaller minority institutions) and the community-based organizations or practices with whom they partner, requiring a conscious discipline of partnership (share the power, share the money, share the data, share interpretation, and share a longterm commitment) to overcome these structural inequities. ${ }^{30}$

\section{Shift Focus from Provider Bebaviors to Systems Change}

Systems change means reengineering the primary care practice to reset the default setting from "don't do anything unless the doctor orders it," to "do automatically the evidence-based thing the doctor would want to have done." In a meta-analysis of interventions designed to improve immunization and cancer screening rates in primary care settings, Stone et al found that many commonly used quality improvement methods were relatively ineffective compared with systems-level or organizational change. $^{31}$

Within the practice, systems change will often mean moving care processes upstream to nurses (via protocols), to front-desk staff (handing out ageand gender-specific patient education materials), and into the waiting room (self-scoring depression scales or cardiovascular risk kiosks). ${ }^{32-35}$ It also will mean using the power of information systems to improve direct patient care (but more than just electronic prompts or reminders). ${ }^{36}$ The frequency of "missed opportunities" in primary care visits is embarrassingly high for disparities-relevant services such as giving adult immunizations, diagnosing depression, or intensifying the regimens of patients with partially controlled hypertension or diabetes. The key is to make good care automatic. ${ }^{37}$ For example, point-of-care testing done by nurses automatically on every patient visit so that the result is on the chart at the moment of the physician visit significantly improves both process measures and glycemic control and potentially reduces practice variation that could contribute to health disparities. ${ }^{38}$ Systems change has similarly been proven to be effective in improving childhood immunization rates in pediatric practice ${ }^{39}$ and depression treatment in primary care settings. ${ }^{40}$

\section{Blur the Boundaries between Community Health and Practice-based Research}

There is an abundance of practice-based research documenting the effectiveness of team approaches to improving quality of care within the primary care practice. However, health disparities have both a medical and a community context. High-risk patients live within a family in a neighborhood, just as the practices that serve them work within the context of structural inequities in a larger health care system.

Therefore, to impact disparities in population health outcomes, interventional disparities research cannot be confined to projects conducted within the clinic walls. This means moving beyond traditional practice-based research by building explicit care-sharing partnerships with public health units and with community-based pharmacists, nurse care managers, behavioral health professionals, hospital nutritionists, physical therapists, etc. Various studies, for example, have shown dramatic improvements in chronic disease outcomes that can be achieved by building bridges between communitybased pharmacists and the primary care practice. ${ }^{41-43}$

The primary care team can extend even further into the community by training community health workers or "promotoras" for specific neighborhoods or subgroups of the community. People from the same culture, neighborhood, and economic level are often much more powerful change agents for health behavior than are physicians or professional health educators, whose role is not eliminated but rather changed to train and support the community health workers. ${ }^{44-46}$ The primary care team can also extend into the community by creating specific venues (health clubs, churches, hair salons/barber shops, Internet cafés, etc) for interacting with people outside of the practice, ${ }^{47,48}$ focusing especially on segments of the community that may never appear in the examination room, ie, those who are currently not focused on their own health (eg, men $)^{49,50}$ or not connected with routine care in a primary care home (eg, the uninsured and underserved). ${ }^{51}$

\section{Measure Community-level, Population Health Outcomes}

Just as interventions to reduce health disparities cannot be confined within the clinic walls, outcome measures must move to the population level as well. 
In other words, disparities reduction research can move beyond measuring process measures on patients within practices to measuring population health outcomes for high-disparity populations at the local-area community level. Many studies have improved documentation of environmental trigger counseling or peak flow testing within a safety net primary care practice, but to reduce disparities, this should ultimately translate into reductions in emergency department visits and hospital admissions for low-income children living within the zip codes served by that health center. Some of the current initiatives in Medicaid disease management have begun to show some improvements in populationlevel health outcomes for specific diseases, but results have been mixed. ${ }^{52,53}$

A better example of improving health outcomes community-wide is a project designed to reduce adverse cardiovascular outcomes in the North Karelia region of Finland. This project was initiated in 1972 in response to requests from the population to address high levels of coronary artery disease. A comprehensive, community-coordinated intervention was developed to address multiple cardiac risk factors. Blood pressures and cholesterol levels declined community-wide, and cardiovascular mortality declined by $75 \%$ over 25 years. ${ }^{54}$ Although cardiovascular mortality declined nationwide in Finland, the improvements in the North Karelia region were significantly greater. ${ }^{55} \mathrm{Al}-$ though the United States has also experienced some similar public health successes for the broader population, there has been to date no specific intervention in the United States that has rigorously demonstrated the ability to reduce health outcome disparities (eg, black/white disparities rate ratios, for example) on a zip code, county, or metro-area level.

\section{Whole-Person Outcomes Amid Complex Co- Morbidities}

Interventions focused on single-disease guidelines are only meaningful for patients with only one disease. In high-disparity populations, co-morbidities abound. Most primary care patients have multiple chronic diseases and risk factors as well as common co-morbid mental health conditions. ${ }^{56-59}$ It is precisely the patients with the most co-morbidities who are likely to contribute the most to disparities in health outcomes. A typical primary care patient has depression, hypertension, hyper- lipidemia, obesity, and diabetes-but reductionistic research models select unrepresentative sets of patients with unnecessary exclusion criteria or target single problems with single interventions. Research designed to reduce or eliminate health disparities must focus on the complex mix of health disparities experienced by individual persons, not just isolated disease states. ${ }^{60}$ In the North Karelia project cited above, the focus on reducing multiple behavioral risk factors all at the same time not only impacted cardiovascular deaths but later demonstrated similar declines in lung cancer deaths as a result of decreased smoking. ${ }^{61}$

\section{Triangulate Interventions on Practice, Patient, and Community}

Traditional experimental study designs test one isolated intervention, holding all other variables constant. Because patients in high-disparity groups are medically complex and their disparities are linked to complex social factors, no single intervention is likely to make a meaningful difference in reducing health disparities. Attacking the complexities of health disparities will require multidimensional, comprehensive interventions at the practice level (in the context of their local health care system). These multidimensional interventions can then triangulate on improved outcomes from at least three directions: the provider, patient, and community.

Satcher and Rust recently published a threedimensional model for reducing disparities in lung health which illustrates this approach. ${ }^{62}$ For example, asthma is a high-disparity condition for which most adverse outcomes are largely preventable. Improving clinical practice to accurately stage patients and provide daily inhaled corticosteroids can significantly reduce hospitalizations. Individual behaviors such as daily peak flow testing and empowered self-management can also significantly improve outcomes. At the systems level, patients who have access to a primary care home, rather than using hospital emergency rooms as their primary source of care, also have significantly better outcomes. Finally, interventions in the home and in the surrounding community can reduce secondary smoke exposure, dust mite and cockroach antigen, and diesel fumes from school buses idling their engines outside the classroom. The National Cooperative Inner City Asthma study is an example of such a multidimensional intervention that triangu- 
lated on the provider in the microsystem of his/her practice and local health care system, the person in the context of his/her family and social networks, and both provider and patient within the larger context of their community. ${ }^{63}$ Similarly, the Forsyth County Cancer Screening Project combined interventions reaching into public health and primary care practices with community outreach interventions and achieved significant increases in both breast and cervical cancer screenings in the target community relative to a control community. ${ }^{64}$

\section{Dynamic versus Static Interventions}

The traditional experimental study design also typically requires that the intervention is held constant throughout the study. A static, unchanging intervention is poorly suited to the complex and even chaotic environments of safety net primary care practices and precludes the opportunity for learning, adaptation, response to feedback, and continuous improvement. Interventions that are adapted to local conditions, respond to changes in the environment, and are continuously refined based on experience and on-going feedback of outcomes measures are much more likely to reduce health disparities. The process, structure, and parameters for such change can still be defined explicitly to allow for rigorous testing and research replicability. In the quality improvement literature, this is often described in terms of repeated iterations of the plan-do-study-act cycle. Interestingly, the Breakthrough Series Collaboratives reported that this multicycle rapid-change model achieved better quality and outcomes improvements in the safety net community health centers than they did in more highly resourced private sector settings. ${ }^{65} \mathrm{Al}-$ though this model is now being replicated in centers across the country in an initiative called the Health Disparities Collaboratives, the primary focus is on quality of care and health outcomes such as glycemic control across the health center's entire patient population (ie, the reduction in disparities will come from universally improving care for a de facto high-disparity patient population, as opposed to reducing disparities rate ratios within the practice). ${ }^{66}$

\section{Rapid Cycle Time}

Disparities research is evolving also from academic cycle time to rapid-change cycle time. A typical grant-funded research project has a 3- to 5-year cycle time to design, implement, evaluate, and dis- seminate one intervention. Bodenheimer et al found that the practice-based research model could often be a barrier to real-world quality improvement, in part because of the slower cycle time generated by Institutional Review Board approval and patient enrollment/informed consent requirements. ${ }^{67}$ In contrast, effective quality improvement programs emphasize rapid-cycle change using plan-do-study-act feedback loops. Disease management programs typically receive weekly or monthly data feeds, which provide rapid-cycle feedback on the effectiveness of current interventions.

Eliminating disparities demands this rapid cycling of measurement, intervention, remeasurement, redesigned interventions, remeasurement, etc. These rapid-cycle feedback loops cannot be "tacked-on" as chart audits or disease registries but must be built into the systems and processes of care at every level. Zhang et al showed that the implementation of a rapid-cycle computerized concurrent data feedback system was a key element in improving in-hospital care for acute myocardial infarction. ${ }^{68}$

Adopting this rapid-change model, the Robert Wood Johnson Foundation "Urgent Matters" program encouraged participating emergency departments to implement at least one systems change per week over the course of a year, resulting in improvements of more than $50 \%$ on specific measures such as waiting time in the emergency department or time from clinical decision to clinical action. ${ }^{69}$ Perhaps similar results could be achieved with rapid-cycle feedback loops between the primary care practice and emergency department to reduce racial/ethnic disparities (or uninsured vs insured disparities) in measures such as emergency department visits for uncontrolled hypertension or diabetes.

\section{Demand Scientific Rigor but Redefine Scientific Rigor to Include New Methods for Measuring Impact in Complex, Dynamic Systems}

Improving disparities in real-world settings is challenging enough, but proving that a specific intervention was responsible requires sufficiently robust research design and analytical models to prove that the interventions can actually reduce health disparities in real-world settings. Although complicated to manage, we have demonstrated that it is possible to conduct a rigorously designed, group randomized controlled trial of interventions across many safety-net primary care practices in multiple states. ${ }^{4}$ 
Additional examples of ongoing projects include a randomized controlled trial with factorial design related to blood pressure adherence in clinics in Baltimore $^{70}$ and a randomized controlled trial of a patient-centered, culture-specific quality improvement program to improve depression care and outcomes in urban, community-based clinics in Delaware and Maryland. ${ }^{71}$

However, the group-randomized controlled trial design, although most scientifically rigorous, is both cumbersome and expensive and unlikely to lead to rapid-cycle testing of enough interventions to significantly reduce or eliminate health disparities. Real-world disparities research will require alternative study designs and statistical methods that have sufficient rigor to test rapidly evolving, multidimensional interventions adapted to local community needs in a cost-effective manner. Some of these methods will include mixed-methods research, time-series analyses of frequent measures, and multilevel or clustered analysis. ${ }^{72,73}$ Recently published books by Blumenthal and Crosby provide an excellent overview of these alternative study designs and analytical methods. ${ }^{74,75}$

Optimal study design is not only driven by research rigor but also by a sense of partnership with communities. For example, a randomized controlled trial of a disparities intervention versus a placebo or sham intervention is more fair to both communities if there is a commitment to replication of beneficial aspects of the program in the control community. Therefore, community-based interventions will often include a crossover design, or phased stages of implementation, to assure benefit to both intervention and control communities.

\section{Test Interventions that Are Replicable, Scalable, and Sustainable in Real-World, Underresourced Settings that Serve High-disparity Populations}

Many translational intervention models are replicable (achievable in highly motivated practices with additional resources) but not scalable (adaptable to all practices through systematic adoption). Scalability once referred primarily to expansion of information technology systems to large numbers of users but is now increasingly being used to describe the programmatic and human dimensions of expanding health programs to scale, ${ }^{76}$ even community-based programs that have no IT component at all. ${ }^{77}$

Even interventions that are tested in real-world settings may not be scalable or sustainable when research grant funding is withdrawn. For example, Love and Spiegel noted that, "although the [inner city asthma project] proved successful, moving from a research design to the real world of implementation was difficult." ${ }^{78}$ Ultimately, real-world reductions in health disparities will require interventions that are not only effective and replicable but scalable and sustainable across most of the diverse practices that serve high-disparity patient populations in real-world settings. For example, a protocol-driven HbA1c point-of-care testing program, which redesigns work flow to make improved care automatic and which balances new tasks with the elimination of inefficiencies or unnecessary work, could be adopted by hundreds of centers simultaneously with limited external stimulus.

\section{Conclusion}

The 12 strategies discussed in this article are not without challenges. Linking interventions to community-level outcomes can be costly. Using multidimensional interventions in real-world settings may reduce replicability because each setting may require new adaptations of the model. Assuring meaningful community partnership (not just participation) can increase costs and lengthen, rather than reduce, cycle times, at least initially.

On the other hand, the bulk of research to date has not significantly reduced disparities, especially at the community or population level. The challenge for disparities researchers in the 21 st century will be to show, at the level of an entire zip code or county, and then an entire state, and ultimately for the nation, that we can substantially reduce disparities not just in quality of care but in health outcomes across all racial/ethnic groups and achieve health equity for all.

\section{References}

1. Levine RS, Foster JE, Fullilove RE, et al. Blackwhite inequalities in mortality and life expectancy, 1933-1999: implications for healthy people 2010. Public Health Rep 2001;116:474-83.

2. van Ryn M, Fu SS. Paved with good intentions: do public health and human service providers contribute to racial/ethnic disparities in health? Am J Public Health 2003;93:248-55.

3. Institute of Medicine. Unequal treatment: confronting racial and ethnic disparities in health care. Washington (DC): National Academies Press; 2003. p. 4.

4. Highlights. In: National Health Care Disparities Report. Rockville (MD): US Department of Health and 
Human Services, Agency for Healthcare Research and Quality; 2005 Dec. Report No.: 06-0017. Available from: http://www.ahrq.gov/qual/nhdr05/ nhdr05.pdf. pp. 2-4.

5. Satcher D, Fryer GE, McCann J, Troutman A, Woolf S, Rust G. What if we were equal? A comparison of the black-white mortality gap in 1960 and 2000. Health Aff (Millwood) 2005;24:459-64.

6. Quality of Health Care. In: National Health Care Disparities Report. Rockville (MD): US Department of Health and Human Services, Agency for Healthcare Research and Quality; 2005 Dec. Report No.: 06-0017. Available from: http://www.ahrq.gov/qual/ nhdr05/nhdr05.pdf. pp. 28-85.

7. Sequist TD, Schneider EC. Addressing racial and ethnic disparities in health care: using federal data to support local programs to eliminate disparities. Health Serv Res 2006;41:1451-68.

8. Giordano TP, Morgan RO, Kramer JR, et al. Is there a race-based disparity in the survival of veterans with HIV? J Gen Intern Med 2006;21:613-7.

9. Rehman SU, Hutchison FN, Hendrix K, Okonofua EC, Egan BM. Ethnic differences in blood pressure control among men at Veterans Affairs clinics and other health care sites. Arch Intern Med 2005;165: 1041-7.

10. Shi L, Stevens GD, Wulu JT Jr, Politzer RM, Xu J. America's health centers: reducing racial and ethnic disparities in perinatal care and birth outcomes. Health Serv Res 2004;39:1881-901.

11. Politzer RM, Yoon J, Shi L, Hughes RG, Regan J, Gaston MH. Inequality in America: the contribution of health centers in reducing and eliminating disparities in access to care. Med Care Res Rev 2001;58: 234-48.

12. Halanych JH, Wang F, Miller DR, Pogach LM, Lin H, Berlowitz DR, Frayne SM. Racial/ethnic differences in diabetes care for older veterans: accounting for dual health system use changes conclusions. Med Care 2006;44:439-45.

13. Gurmankin AD, Polsky D, Volpp KG. Accounting for apparent "reverse" racial disparities in Department of Veterans Affairs (VA)-based medical care: influence of out-of-VA care. Am J Public Health 2004;94:2076-8.

14. Mold JW, Peterson KA. Primary care practice-based research networks: working at the interface between research and quality improvement. Ann Fam Med 2005;3 Suppl 1:S12-20.

15. Schwenk TL, Green LA. The Michigan Clinical Research Collaboratory: following the NIH Roadmap to the community. Ann Fam Med 2006;4 Suppl 1:S49-54; discussion S58-60.

16. Daniels EC, Bacon J, Denisio S, et al. Translation squared: a group-randomized controlled trial of an intervention to improve asthma care in safety net primary care settings. J Asthma 2005;42:499-505.
17. Satcher D, Rust G. Achieving health equity in America. Ethn Dis 2006;16(2 Suppl 3):S3-8-13.

18. Lemon SC, Zapka JG, Estabrook B, Benjamin E. Challenges to research in urban community health centers. Am J Public Health 2006;96:626-8.

19. Rabinowitz HK, Becker JA, Gregory ND, Wender RC. NIH funding in family medicine: an analysis of 2003 awards. Ann Fam Med 2006;4:437-42.

20. Beach MC, Gary TL, Price EG, et al. Improving health care quality for racial/ethnic minorities: a systematic review of the best evidence regarding provider and organization interventions. BMC Public Health 2006;6:104.

21. Lillie-Blanton M, Anthony JC, Schuster CR. Probing the meaning of racial/ethnic group comparisons in crack cocaine smoking. JAMA 1993;269:993-7.

22. Burd S. Racial imbalance found in NIH grants. Chronicle of Higher Education. 1993 Nov 10;40: A28.

23. Shavers VL, Fagan P, Lawrence D, et al. Barriers to racial/ethnic minority application and competition for NIH research funding. J Natl Med Assoc 2005; 97:1063-77.

24. Census Briefs 2000: Overview of Race and Hispanic Origin. Washington (DC): US Census Bureau; 2001. p. 3.

25. Minorities in medical education: facts \& figures 2005. Washington (DC): Association of American Medical Colleges; 2005 Spring. p. 30. Available from: https:// services.aamc.org/Publications/showfile.cfm? file $=$ version53.pdf\&prd_id=133\&prv_id=154\&pdf_id $=53$.

26. Diversity in the physician workforce: facts \& figures 2006. Washington (DC): Association of American Medical Colleges; 2006 Summer. Available from: https:// services.aamc.org/Publications/showfile.cfm? file $=$ version74.pdf\&prd_id $=161 \&$ prv_id $=191 \&$ pdf id $=74$.

27. Bach PB, Pham HH, Schrag D, Tate RC, Hargraves JL. Primary care physicians who treat blacks and whites. N Engl J Med 2004;351:575-84.

28. Zerhouni EA. Translational and clinical sciencetime for a new vision. N Engl J Med 2005;353: 1621-3.

29. Wallerstein NB, Duran B. Using community-based participatory research to address health disparities. Health Promot Pract 2006;7:312-23.

30. Blumenthal DS. A community coalition board creates a set of values for community-based research. Prev Chronic Dis 2006;3:A16.

31. Stone EG, Morton SC, Hulscher ME, et al. Interventions that increase use of adult immunization and cancer screening services: a meta-analysis. Ann Intern Med 2002;136:641-51.

32. Fowler J, Hardy J, Howarth T. Trialing collaborative nursing Models of Care: the impact of change. Aust J Adv Nurs 2006;23:40-6.

33. Rust G, Strothers HS 3rd, Zimmerman RK. Reengineering the primary care practice to eliminate 
adult immunization disparities. Ethn Dis 2005;15(2 Suppl 3):S3-21-S3-6.

34. Couch C, Sheffield P, Gerthoffer T, Ries A, Hollander P. Clinical outcomes in patients with type 2 diabetes managed by a diabetes resource nurse in a primary care practice. Proc (Bayl Univ Med Cent) 2003;16:336-40.

35. Reiss-Brennan B, Briot P, Cannon W, James B. Mental health integration: rethinking practitioner roles in the treatment of depression: the specialist, primary care physicians, and the practice nurse. Ethn Dis 2006;16(2 Suppl 3):S3-37-43.

36. O'Connor PJ, Crain AL, Rush WA, Sperl-Hillen JM, Gutenkauf JJ, Duncan JE. Impact of an electronic medical record on diabetes quality of care. Ann Fam Med 2005;3:300-6.

37. Bodenheimer T, Wagner EH, Grumbach K. Improving primary care for patients with chronic illness. JAMA 2002;288:1775-9.

38. Miller CD, Barnes CS, Phillips LS, et al. Rapid HbA1c availability improves clinical decision-making in an urban primary care clinic. Diabetes Care 2003;26:1158-63.

39. Bordley WC, Margolis PA, Stuart J, Lannon C, Keyes L. Improving preventive service delivery through office systems. Pediatrics 2001;108:E41.

40. Dietrich AJ, Oxman TE, Williams JW Jr, Kroenke K, Schulberg HC, Bruce M, Barry SL. Going to scale: re-engineering systems for primary care treatment of depression. Ann Fam Med 2004;2:301-4.

41. McLean W, Gillis J, Waller R. The BC Community Pharmacy Asthma Study: a study of clinical, economic and holistic outcomes influenced by an asthma care protocol provided by specially trained community pharmacists in British Columbia. Can Respir J 2003;10:195-202.

42. Kiel PJ, McCord AD. Pharmacist impact on clinical outcomes in a diabetes disease management program via collaborative practice. Ann Pharmacother 2005; 39:1828-32.

43. Lee SS, Cheung PY, Chow MS. Benefits of individualized counseling by the pharmacist on the treatment outcomes of hyperlipidemia in Hong Kong. J Clin Pharmacol 2004;44:632-9.

44. Norris SL, Chowdhury FM, Van Le K, et al. Effectiveness of community health workers in the care of persons with diabetes. Diabet Med 2006;23:544-56.

45. Hunter JB, de Zapien JG, Papenfuss M, Fernandez ML, Meister J, Giuliano AR. The impact of a promotora on increasing routine chronic disease prevention among women aged 40 and older at the US-Mexico border. Health Educ Behav 2004;31(4 Suppl):18S-28S.

46. Larkey L. Las mujeres saludables: reaching Latinas for breast, cervical and colorectal cancer prevention and screening. J Community Health 2006;31:69-77.

47. Eng E, Smith J. Natural helping functions of lay health advisors in breast cancer education. Breast Cancer Res Treat 1995;35:23-9.

48. Gesler WM, Arcury TA, Skelly AH, Nash S, Soward A, Dougherty M. Identifying diabetes knowledge network nodes as sites for a diabetes prevention program. Health Place 2006;12:449-64.

49. Treadwell HM, Ro M. Poverty, race, and the invisible men. Am J Public Health 2003;93:705-7.

50. Williams DR. The health of men: structured inequalities and opportunities. Am J Public Health 2003;93:724-31.

51. Grumbach K, Bodenheimer T. A primary care home for Americans: putting the house in order. JAMA 2002;288:889-93.

52. Rossiter LF, Whitehurst-Cook MY, Small RE, et al. The impact of disease management on outcomes and cost of care: a study of low-income asthma patients. Inquiry 2000;37:188-202.

53. Villagra V. Strategies to control costs and quality: a focus on outcomes research for disease management. Med Care 2004;42(4 Suppl):III24-30.

54. Nissinen A, Kastarinen M, Tuomilehto J. Community control of hypertension-experiences from Finland. J Hum Hypertens 2004;18:553-6.

55. Puska P, Vartiainen E, Tuomilehto J, Salomaa V, Nissinen A. Changes in premature deaths in Finland: successful long-term prevention of cardiovascular diseases. Bull World Health Organ 1998;76:419-25.

56. Ortega AN, Feldman JM, Canino G, Steinman K, Alegria M. Co-occurrence of mental and physical illness in US Latinos. Soc Psychiatry Psychiatr Epidemiol. Epub 2006 Sept 29.

57. Rutledge T, Reis SE, Olson MB, et al. Depression symptom severity and reported treatment history in the prediction of cardiac risk in women with suspected myocardial ischemia: the NHLBI-sponsored WISE study. Arch Gen Psychiatry 2006;63:874-80.

58. Lang CC, Mancini DM. NonCardiac comorbidities in heart failure. Heart. Epub 2006 Feb 17.

59. Kato K, Sullivan PF, Evengard B, Pedersen NL. Chronic widespread pain and its comorbidities: a population-based study. Arch Intern Med 2006;166: 1649-54.

60. Fortin M, Dionne J, Pinho G, Gignac J, Almirall J, Lapointe L. Randomized controlled trials: do they have external validity for patients with multiple comorbidities? Ann Fam Med 2006;4:104-8.

61. Puska P, Korhonen HJ, Torppa J, Tuomilehto J, Vartiainen E, Pietinen P, Nissinen A. Does community-wide prevention of cardiovascular diseases influence cancer mortality? Eur J Cancer Prev 1993;2: 457-60.

62. Satcher D, Rust G. A three-dimensional approach to the elimination of racial-ethnic disparities in lung health. Clin Chest Med 2006;27:535-42, viii.

63. Spiegel J, Love AS, Wood PR, Griffith M, Taylor KR, Williams SG, Redd SC. The Inner-City Asthma Intervention: description of a community-based im- 
plementation of an evidence-based approach to asthma management. Ann Allergy Asthma Immunol 2006;97(1 Suppl 1):S6-10.

64. Paskett ED, Tatum CM, D'Agostino R Jr, et al. Community-based interventions to improve breast and cervical cancer screening: results of the Forsyth County Cancer Screening (FoCaS) Project. Cancer Epidemiol Biomarkers Prev 1999;8:453-9.

65. Wagner EH, Glasgow RE, Davis C, Bonomi AE, Provost L, McCulloch D, Carver P, Sixta C. Quality improvement in chronic illness care: a collaborative approach. Jt Comm J Qual Improv 2001;27:63-80.

66. Wang A, Wolf M, Carlyle R, Wilkerson J, Porterfield D, Reaves J. The North Carolina experience with the diabetes health disparities collaboratives. Jt Comm J Qual Saf 2004;30:396-404.

67. Bodenheimer T, Young DM, MacGregor K, Holtrop JS. Practice-based research in primary care: facilitator of, or barrier to, practice improvement? Ann Fam Med 2005;3 Suppl 2:S28-32.

68. Zhang H, Alexander JA, Luttrell J, O'Connor GT, Daley J, Paris M. Data feedback and clinical process improvement in acute myocardial infarction. Am Heart J 2005;149:856-61.

69. Wilson M, Nguyen K. Bursting at the seams: improving patient flow to help America's hospitals. Washington (DC): Urgent Matters, The George Washington University Medical Center; 2004 Sep. Available from: http://www.urgentmatters.org/ reports/UM_WhitePaper_BurstingAtTheSeams.pdf.

70. Patient-Physician Partnership to Improve High Blood Pressure Adherence. Indexed at clinicaltrials.gov. National Library of Medicine. Accessed at http://clinicaltrials.gov/show/NCT00123045, on July 18, 2006.
71. Patient-Centered Depression Care for African Americans. Indexed at clinicaltrials.gov. National Library of Medicine. Accessed at http://clinicaltrials. gov/ct/show/NCT00243425? order=1 on July 18, 2006.

72. Shi, L. Experimental Research. In: Health Services Research Methods. Albany (NY): Delmar Publishers, Inc; 1997.

73. Cooper LA, Hill MN, Powe NR. Designing and evaluating interventions to eliminate racial and ethnic disparities in health care. J Gen Intern Med 2002;17:477-86.

74. Blumenthal DS, DiCLemente RJ, editors. Community-based health research. New York: Springer; 2006.

75. Crosby A, DiClemente RJ, Salazar LF, editors. Research methods in health promotion. San Francisco: Jossey-Bass; 2006.

76. Pronk NP, Boucher JL, Gehling E, Boyle RG, Jeffery RW. A platform for population-based weight management: description of a health plan-based integrated systems approach. Am J Manag Care 2002; 8:847-57.

77. Opul D, Chang HE, Bukare BO, Opio A, Masters H, Weinstein $M$. Towards replicable and scalable AIDS treatment models for resource-constrained settings. 14th International AIDS Conference; 2002 Jul 7-12; Barcelona, Spain; abstract no. F12305. Available from: http://www.aegis.com/conferences/iac/2002/ F12305.html.

78. Love AS, Spiegel J. The Inner-City Asthma Intervention tool kit: best practices and lessons learned. Ann Allergy Asthma Immunol 2006;97(1 Suppl 1): S36-9. 